\title{
Body Type Classification of the Three-dimensional Torso Shape of Japanese Men Aged 20 to 70 Years for Efficient Clothing Design
}

\author{
Keiko WATANABE* \\ Kyoto Women's University, Kyoto, Japan \\ DOI: $10.15221 / 17.347$ http://dx.doi.org/10.15221/17.347
}

\begin{abstract}
In Japan, the men's clothing industry has faced repeated price competition. However, it seems that price competition is also reaching its limit. Consumers are looking for new added value, and clothes that fit the body by a new made-to-measure system could be one solution. An appropriate classification system of body types is needed for an efficient made-to-measure system. However, analysis of male bodies has rarely been performed in Japan. The final purposes are to classify the body types among Japanese men, and create a new made-to-measure system using three-dimensional body scanning data. In this study, our aims were to clarify factors that account for the diversity among Japanese men's body shapes, and to develop a classification system for Japanese men's body shapes. The data of 50 men in each age group of men in their 20s, 30s, 40 s, and 50 s and 50 men in their 60 s and 70 s, totaling 250 men, were randomly selected from 429 men aged 20 to 79 years old. A body line scanner was used to measure each person's body. In this study, the trunk, which was restricted to the inside of the arm hole, below the neckline, and above the trochanter point, was analyzed. The origin was unified based on the landmarks: the back-neck point was the origin of the X-axis (transverse direction), the trochanter point was the origin of the $\mathrm{Y}$-axis (vertical direction) and the right-side neck point was the origin of the Z-axis (sagittal direction). To enable statistical analysis, the data from each individual were transformed to a homologous model by HBM software. The homologous models were analyzed by principal component analysis with DHRC-HBS Human Body Shape Statistics software. Seven factors were extracted and interpreted as follows. The first principal component (PC1) was the factor of height of the trunk. PC2 was inclination of the torso forward or backward above the waistline. PC3 was thickness of the torso. PC4 and PC5 were left / right difference. PC6 was forward or backward shoulder. PC7 was considered as distortion of the shoulder relative to the lower trunk. The principal component scores for PC1, PC2, PC3, and PC6 were used in cluster analysis with the statistics software IBM SPSS Statistics. The analysis resulted in seven clusters. CL1 and CL4 were similar to the average shape of the 250 subjects, although CL4 was taller than CL1. CL1 and CL4 accounted for $50 \%$ of the men in the groups of men in their 30s, 40s, and 50s. CL6 was slenderer than CL1; in CL6, the body trunk was thin and showed swayback, and its frequency was relatively high among men in their 20s. CL7 represented good posture with a developed chest. CL3 had a round back and front shoulder and these men were short; CL3 comprised $50 \%$ or more of the men in their 60 s and 70 s. CL2 was bending backward with a protruded stomach, which is considered to be the so-called metabolism type, and comprised $20 \%$ of men in their 50s. This body classification system is considered to be applicable for virtual dress dummy design and for a made-to-measure system.
\end{abstract}

Keywords: dress dummy, principal component analysis, classification of body type, pattern alteration

\section{Introduction}

In recent years, clothing items cannot be sold at regular prices, and it is becoming more common to sell them at lower prices on sale in Japan. According to the Ministry of Economy, Trade and Industry of Japan, the purchase unit price index for clothing in 2013 fell to about $60 \%$ of that in 1991 [1]. In addition, the size of the clothing market has decreased from about 15.3 trillion yen per year to about 10.5 trillion yen per year. The men's clothing industry especially for suits has faced repeated low price competition. People in a wide range of occupations in Japan have been wearing suits on a daily basis like uniforms. Therefore, being low-priced is a very important factor in purchase decision-making. In recent years, it seems that price competition is also reaching its limit and consumers are looking for new added value. The ready-made garment industry for men emphasizes sewing techniques that imitate bespoke tailoring and mobility comfort in their advertisements. The major mass merchandising stores have started to customize size, fabric and details.

In a questionnaire survey distributed to consumers and Japanese companies in $2007,81 \%$ of consumers replied that ready-to-wear clothes do not fit their body, and $75 \%$ of consumers and apparel manufacturers were interested in mass-customized clothing in Japan [2]. Made-to-measure clothing businesses which are called "size order" or "pattern order" in Japan, in which sizes such as sleeve 
length and waist girth are adjusted and simple pattern alteration is done, are becoming popular in Japan. The sizes are measured by salespeople, but their skill in size measurement is not sufficient, which may cause problems in their products. On the other hand, measurements such as length and girth can be calculated from three-dimensional body scanning data. The measurements can then be used to develop customized patterns to fit individual customers [3]. This may solve the problem of measurement error arising from the low skill of salespeople. However, these methods cannot always accommodate the shape of the pattern to the customer's body shape like made-to-order clothes made by bespoke tailors. However, consumers are seeking suits, coats or dresses with more personalized pattern alterations. Making clothes that fit the particular customer's body by a new made-to-measure system could be one solution. Apparel fit is important not only in terms of comfort, but also aesthetically.

The technology that can automatically accommodate the clothing pattern to the customer's body size and shape, called "body type grade", using apparel computer-assisted design (CAD) systems, is used in many companies in Japan. In the case of size grade in the apparel CAD system, the apexes or specific points of measurement for each pattern piece are defined as grade points. Grade rules are applied to the grade point, which will increase or decrease the measurement of a specific pattern piece at a specified point. Grade rules determine which grade points are moved, and how much the points are moved along the $x$ - and $y$-axes. The same CAD system is used to accommodate "body type grade", in which some specific grade points are moved along the $x$ - and $y$-axes to match the shape of the individual. Also, some specific grade points are moved in the $x$ and $y$ directions to match the body's shape such as sloping/square shoulders or a stooped/flatter back. However, this is not sufficient.

In the author's previous study, the relationships between the principal components of the torso shape and the shape of the body surface of female subjects were observed in terms of clothing alteration or "body type grade" [4]. If the pattern is perfectly adjusted with multiple short measurement values, the shape of the pattern would change. A large change in the shape of the pattern would result in major changes in the silhouette and image that customers cannot accept when it is worn, and would also cause the problem that it cannot be sewn in a factory. For these reasons, the range in which patterns can be altered is limited in the current system.

As a result, the author thought that an appropriate classification system of body types is needed to develop an efficient made-to-measure system. If human body shapes were analyzed and classified into several body type groups, then master patterns could be made for each group and one of the patterns would fit each customer with limited grade point movement to alter it. Few studies have analyzed Japanese men's body shape using three-dimensional data. In this study, 429 men were measured by a three-dimensional body scanner. The aims of the present study were to clarify factors that contribute to the diversity among Japanese men's body shapes, and to establish an efficient body type classification system.

\section{Methods}

\subsection{Subjects and measurement}

Measurements were made in 429 healthy Japanese men aged 20 to 79 years. In each person, 67 items were measured using a Martin style height meter and tape measure, according to the method of Japanese Industrial Standard L-0111 [5]. Then, eighteen landmarks were pasted on the body surface of the person. The neck base line, armhole line and shoulder seam line were drawn on the skin with eyeliner, and the waist line was marked with $3 \mathrm{~mm}$-wide tape. The body was measured using the noncontact three-dimensional scanner named Body Line Scanner (Hamamatsu Photonics, Shizuoka, Japan). The person was asked to maintain a natural standing position, with his feet $20 \mathrm{~cm}$ apart from each other and his arms abducing approximately 20 degrees. The straight line connecting the left and right heels of the subject is strictly aligned parallel to the X-axis. Fifty men in each age group of men in their $20 \mathrm{~s}, 30 \mathrm{~s}, 40 \mathrm{~s}$, and $50 \mathrm{~s}$ and 50 men in their 60 s and $70 \mathrm{~s}$, totaling 250 men, were randomly selected for further analysis to eliminate bias due to differences in the number of men. The major body measurements of the 250 subjects are summarized in Table 1.

\subsection{Analysis of 3D body shape}

To enable statistical analysis, the data for an individual were transformed to a homologous model by the Homologous Body Modeling (HBM) software (Digital Human Research Center, Tokyo, Japan). Homologous modeling is a reconstruction of data points in such a way that the data for each subject will consist of the same number of data points of the same topology, and each data point will have the same anatomical position across the subjects. The origin was unified based on the following landmarks; the back neck point was the origin of the $\mathrm{X}$-axis (transverse direction), the trochanter point was the origin of the $\mathrm{Y}$-axis (vertical direction), and the right side neck point was the origin of the Z-axis (sagittal 
direction). The 3D body data were not rotated around any axis. The areas outside of the armhole line, above the neckline and below the hipline were cut off to analyze the shape of the trunk. Then, 28 landmarks were used as clues to associate the data. The generic model was applied to the body of each subject, and 250 homologous models were created (Fig. 1). These models were then analyzed with principal component analysis using HBS-PCA (Human Body Statistica, Digital Human Research Center, Tokyo, Japan) [6] [7] to clarify which body parts showed major differences among the individuals. The average +2 standard deviations (S.D.) model and average - 2 S.D. model for each principal component were generated by the HBS software. They were visualized and compared to each other to identify the meanings of the principal components. Then, cluster analysis with the nearest neighbor method was performed using the scores of the four principal components by SPSS statistics software (IBM, U.S.A), to classify the body shapes of Japanese men. The average shape of each cluster was calculated and visualized. In addition, the basic statistics of body measurements of each cluster were compared to clarify the characteristics of each cluster.

Table1 The body measurements of the subjects

\begin{tabular}{|c|c|c|c|c|c|c|c|c|c|c|}
\hline \multirow[b]{3}{*}{ Measurement Item } & \multicolumn{10}{|c|}{ Age group (years) } \\
\hline & \multicolumn{2}{|c|}{20 's } & \multicolumn{2}{|c|}{30 's } & \multicolumn{2}{|c|}{40 's } & \multicolumn{2}{|c|}{50 's } & \multicolumn{2}{|c|}{ 60/70's } \\
\hline & Mean & S.D. & Mean & S.D. & Mean & S.D. & Mean & S.D. & Mean & S.D. \\
\hline & $\mathrm{cm}$ & $\overline{\mathrm{cm}}$ & $\mathrm{cm}$ & $\mathrm{cm}$ & $\mathrm{cm}$ & $\mathrm{cm}$ & $\mathrm{cm}$ & $\overline{\mathrm{cm}}$ & $\mathrm{cm}$ & $\mathrm{cm}$ \\
\hline Height & 171.9 & 5.6 & 173.2 & 6.0 & 170.9 & 6.3 & 169.0 & 5.5 & 163.6 & 6.3 \\
\hline Chest girth & 91.0 & 5.2 & 94.8 & 6.0 & 97.3 & 6.1 & 96.0 & 6.4 & 91.3 & 5.3 \\
\hline Waist girth & 76.9 & 5.9 & 82.5 & 7.6 & 85.3 & 8.7 & 85.2 & 8.4 & 85.8 & 8.5 \\
\hline Abdomen girth & 78.4 & 5.3 & 83.5 & 7.1 & 86.3 & 8.3 & 86.5 & 7.5 & 89.5 & 6.1 \\
\hline Hip girth & 92.0 & 4.7 & 95.5 & 5.2 & 96.1 & 5.4 & 94.5 & 5.7 & 90.8 & 4.5 \\
\hline Upper arm girth & 28.9 & 2.6 & 30.5 & 2.5 & 31.2 & 2.3 & 30.6 & 3.3 & 28.9 & 2.8 \\
\hline Thigh girth & 53.6 & 4.4 & 55.6 & 3.9 & 55.9 & 3.9 & 54.2 & 3.8 & 50.3 & 3.8 \\
\hline Shoulder length & 42.9 & 2.2 & 43.9 & 2.1 & 43.3 & 2.8 & 42.5 & 2.2 & 41.2 & 2.6 \\
\hline Shoulder seam & 13.8 & 0.9 & 13.6 & 0.9 & 13.3 & 0.9 & 13.1 & 0.8 & 13.3 & 1.1 \\
\hline Sleeve length & 57.3 & 2.9 & 57.4 & 2.6 & 56.7 & 2.2 & 56.2 & 2.3 & 55.4 & 2.5 \\
\hline Waist length & 47.8 & 2.7 & 48.5 & 2.7 & 49.0 & 3.1 & 48.4 & 2.8 & 45.6 & 2.6 \\
\hline Front waist length & 42.3 & 2.8 & 43.6 & 4.0 & 44.9 & 5.0 & 45.1 & 4.2 & 39.4 & 5.7 \\
\hline \multirow[t]{2}{*}{ Shoulder slope } & 21.6 & 4.2 & 21.2 & 3.7 & 20.1 & 3.5 & 19.9 & 4.1 & 19.2 & 4.6 \\
\hline & $\mathrm{kg}$ & $\mathrm{kg}$ & $\mathrm{kg}$ & $\mathrm{kg}$ & $\mathrm{kg}$ & $\mathrm{kg}$ & $\mathrm{kg}$ & $\mathrm{kg}$ & $\mathrm{kg}$ & $\mathrm{kg}$ \\
\hline Body weight & 62.0 & 7.3 & 68.1 & 10.1 & 70.3 & 10.2 & 67.7 & 9.6 & 61.6 & 8.3 \\
\hline
\end{tabular}

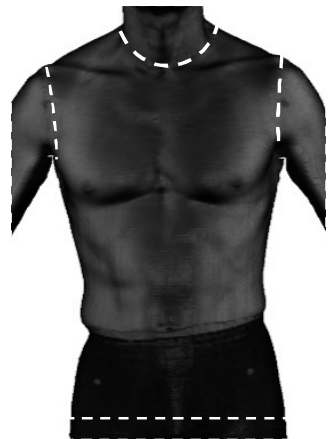

(a)

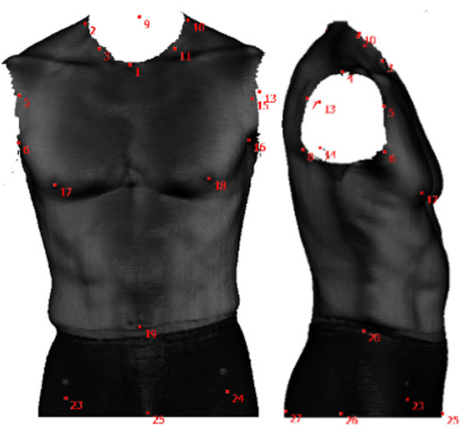

(b)

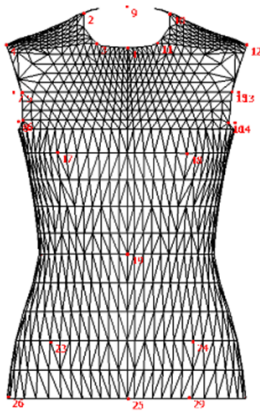

(c)
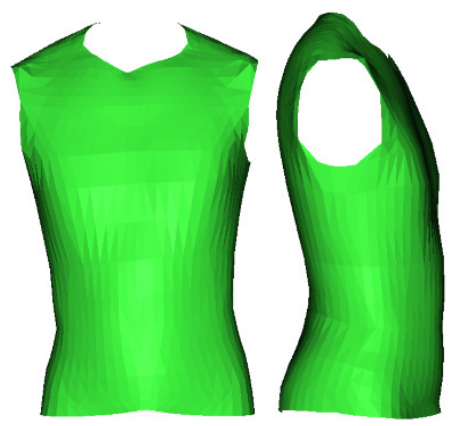

(d)

Fig. 1. The polygon data of a subject (a) after cutting off the unnecessary areas for analysis (b), the generic mode (c) and Homologous model of the same subject (d) are shown. 


\section{Results}

\subsection{Principal component analysis of 3D body shape}

Wide variations in the shape of the torsos of the men were seen. The homologous models of the 250 subjects were analyzed by principal component analysis. Seven principal components with a contribution rate exceeding $4 \%$ were extracted, and the principal component scores for each individual were calculated. The results of the analysis are shown in Table 2. For each principal component, the subjects with a low principal component score and those with a high principal component score were compared. Furthermore, the average +2 S.D. model and average -2 S.D. model for each principal component were generated by the HBS software; they were compared and the principal components were interpreted (Fig. 2). The first principal component (PC1) was interpreted as the height from the trochanter point to the cervical point and aging of body shape. However, the subjects with a higher PC1 principal component score had a clearly shorter torso, a relatively rounded back and flatter hip. As to the first principal component score, the appearance ratio changed with aging. The PC1 principal component score of subjects who had a positive score increased with age, and scores over 60 were seen in the $60 \mathrm{~s} / 70 \mathrm{~s}$ age group (Fig. 3). The older men tended to have high scores and the younger men tended to have lower scores. In our previous study on Japanese women, PC1 was interpreted as the factor of aging of body shape and height of the torso [8]. The silhouettes were clearly different from each other; the female subjects with a low PC1 principal component score had a rounded back and flatter and lower bust, and those with a high PC1 principal component score had a straight back and higher bust. In the present study, the front and lateral silhouette differences between the $\pm 2 S$.D. models were not as clear as those in women. In previous research, it was clear that changes in the lateral silhouette of the body occur more slowly in men than in women [9]. The effect of aging may be more apparent in the female body shape due to the effects of muscle weakness and osteoporosis.

The following principal components were interpreted as follows. The second principal component (PC2) in the men was a factor showing inclination of the torso forward or backward above the waistline level. The neckline of the subjects with a higher PC2 principal component score was located more forward than the neckline of those with a lower score. The third principal component (PC3) was interpreted as thickness of the torso. For PC3, the subjects with a higher principal component score had a thick torso and erect back. On the other hand, the subjects with a lower principal component score had a thin torso and curved back.

The fourth principal component (PC4) was left / right difference, in which the torso was inclined towards the right side or left side. There was no relationship between inclination to the right/left side and age, and it seemed that this was related to habitual things, by nature, and so on.

The fifth principal component (PC5) was interpreted as "the difference between the left and right shoulder inclinations". Observing the two $\pm 2 S$.D. wireframe models from the front, the height of the shoulder tip was different. The sixth principal component (PC6) was posture and forward or backward shoulder. The subjects with a higher PC6 principal component score had an erect posture and the shoulder point and armhole of subjects were located backward.

The seventh principal component (PC7) was considered as distortion of the shoulder relative to the lower trunk. This kind of distortion was seen among the young men and not only in the elderly. PC4, PC5 and PC7 represented distortion or asymmetry of the body. In some previous research studies, the homologous models were rotated to make them have uniform posture. However, in this study, they were not rotated at all, because garments hang down parallel to gravity and it is very important to consider the effect of gravity in clothing design. Gravity causes the center and side seams to hang perpendicular to the floor. Such problems cannot be ignored and it is necessary to discuss how to deal with the design at another opportunity.

Table 2. The result of principal component analysis of the torso of 250 men

\begin{tabular}{rrrrr}
\hline \multirow{2}{*}{ Principal Component } & Eigen value & & Contribution ratio (\%) & Acumlate contribution ratio (\%) \\
\cline { 2 - 4 } & PC1 & 979.0 & 23.5 & 23.5 \\
PC2 & 651.8 & 15.6 & 39.1 \\
PC3 & 505.0 & 12.1 & 51.2 \\
PC4 & 382.3 & 9.2 & 60.4 \\
PC5 & 264.0 & 6.3 & 66.7 \\
PC6 & 198.7 & 4.8 & 71.5 \\
PC7 & 186.7 & 4.5 & 76.0 \\
PC8 & 154.9 & 3.7 & 79.7 \\
\hline
\end{tabular}


PC1
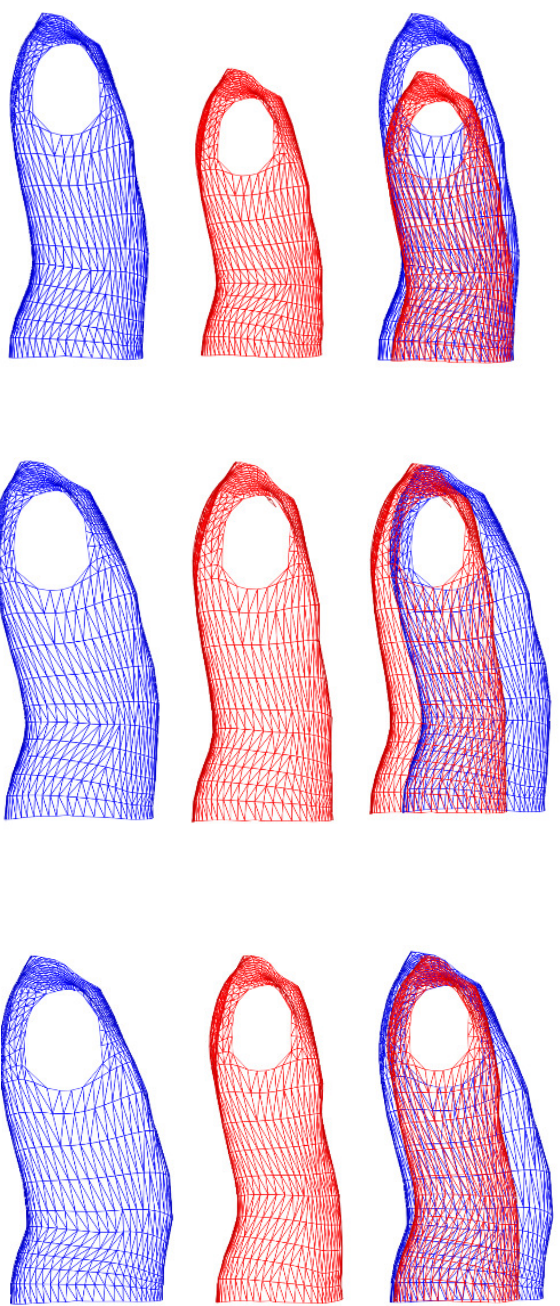

PC3

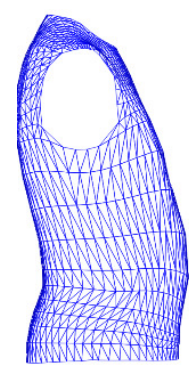

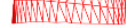
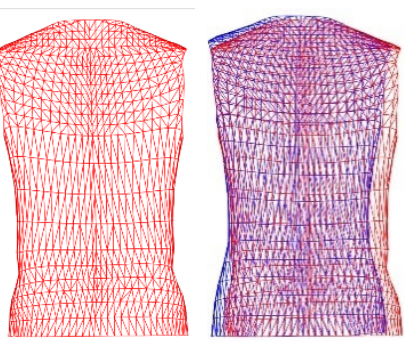

PC7
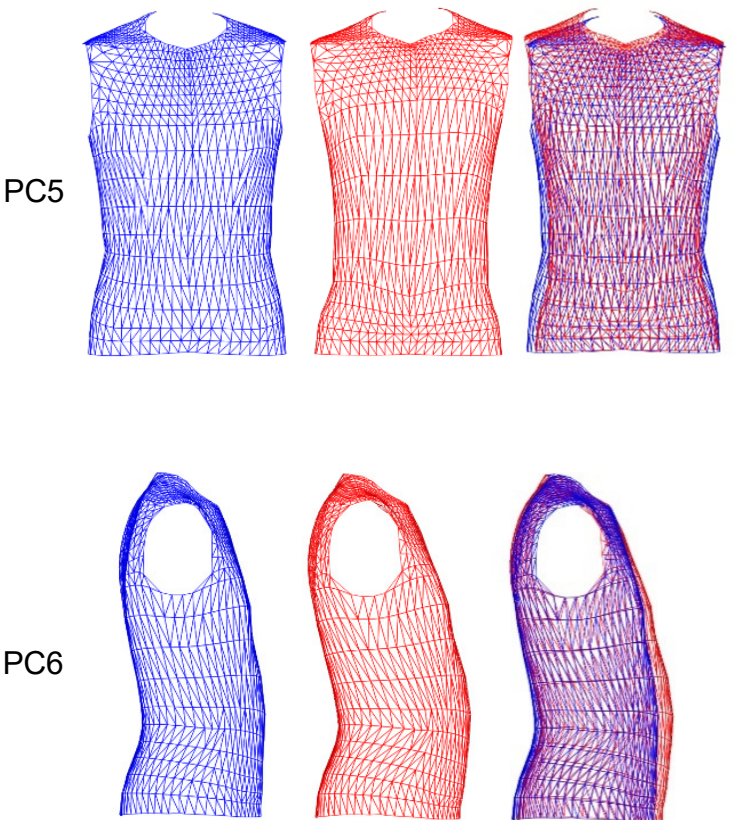

PC6

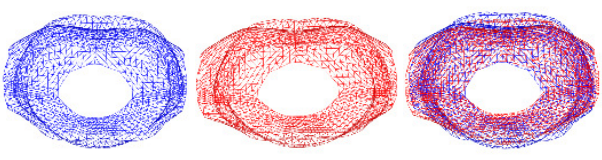

-2S.D. $\quad$ +2S.D. superimposed

+2S.D.

superimposed

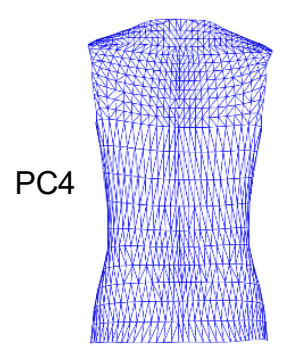

-2S.D.

t2S.D. superimposed

Fig. 2. The average -2S.D. and average +2S.D. models for each principal component, which were generated by HBS 


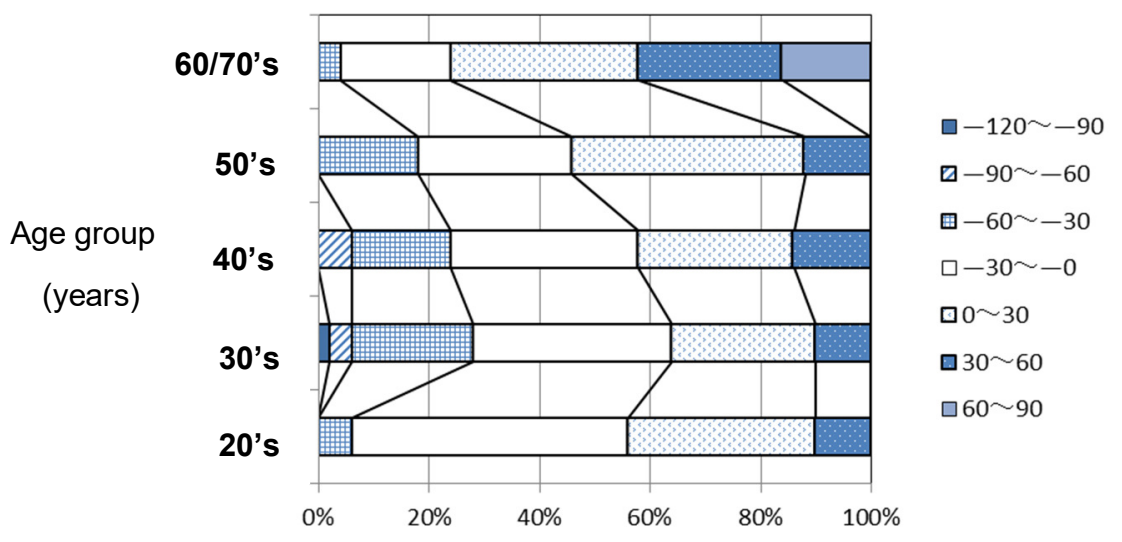

Fig. 3. The frequency of PC1 principal component scores by age group

\subsection{Cluster analysis of 3D body shape}

The principal component scores for PC1, PC2, PC3, and PC6 were used as data in cluster analysis using SPSS statistics software. The principal components related to distortion were excluded from this analysis. Fig. 4 shows the dendrogram using the nearest neighbor method. At several levels of the dendrogram, the subjects were divided into clusters, and the average shape of the subjects in each cluster was calculated and compared. As a result, the 250 subjects were classified into seven clusters, taking into consideration that the difference between clusters was clear. It was thought that the results of this cluster analysis would be most effective for ready-made garments and made-to-measure systems.

Cluster analyses were also done using Ward's method and the k-means method. The results of Ward's method were similar to the results of the nearest neighbor method. The k-mean's method resulted in one large cluster and several small clusters, indicating that bias occurred. Therefore, the nearest neighbor method was chosen.

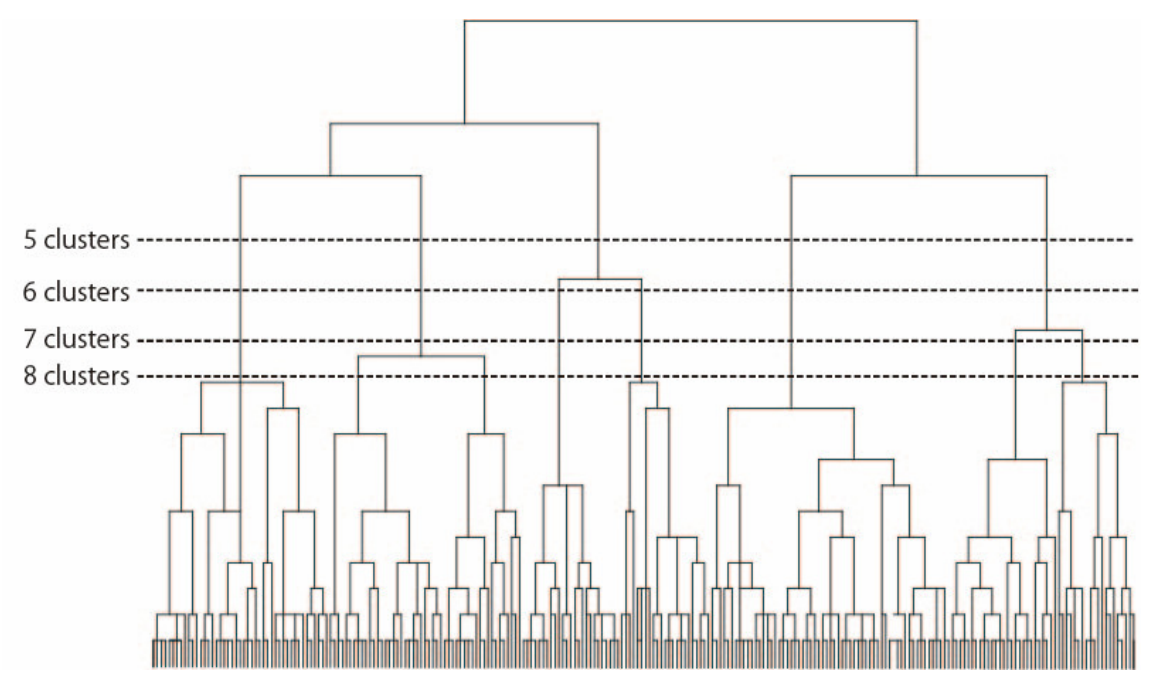

Fig.4 The dendrogram showing the results of cluster analysis using the nearest neighbor method. At 4 levels of the dendrogram, the subjects were divided into clusters, and the average shape of the subjects in each cluster was calculated and compared. 
Fig. 5 shows the average shape of the subjects in the 7 clusters. Fig. 6 shows the average model of all 250 subjects. In order to clarify the characteristics of the 7 clusters, the shapes of the subjects in the seven clusters were compared with the average shape of all 250 subjects, and the mean values and standard deviations of the body measurements of the subjects in the seven clusters were compared with each other (Table 3). To clarify the relationship between age and body type, the frequency of occurrence of each cluster was calculated for each age group (Fig. 7). Cluster 1 (CL1) and cluster 4 (CL4) were similar to the average shape of the 250 subjects, although CL4 was taller than CL1. CL1 and CL4 accounted for $28 \%$ of men in their 20 s, about $40 \%$ in the groups of men in their 30 s, 40 s, and 50 s, and $18 \%$ of men in their $60 / 70$ s. It can be said that CL1 and CL4 are the average body type seen in any age group. Cluster 6 (CL6) and Cluster 7 (CL7) were similar in that the waist was thin. CL6 was slenderer than CL7; in CL6, there was swayback and front shoulder. Their chest width was relatively small compared with the dorsal width. The frequency of CL6 was relatively high among men in their

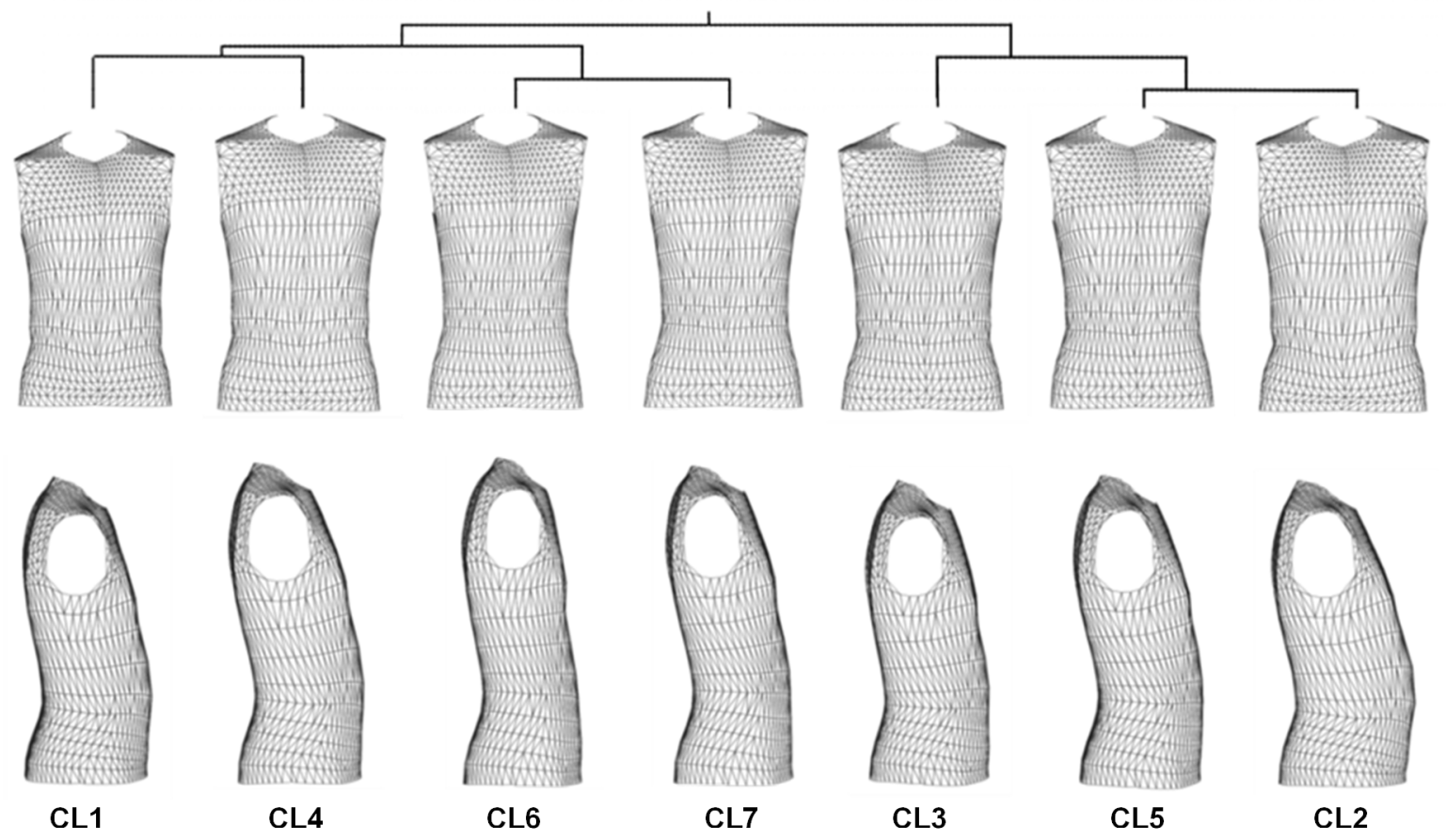

Fig. 5 The average shape of the 7 clusters in which the 250 subjects classified

Table3 The body measurements of the subjects of the 7 clusters

\begin{tabular}{|c|c|c|c|c|c|c|c|c|c|c|c|c|c|c|}
\hline \multirow[b]{2}{*}{ Measurement Item } & \multicolumn{2}{|c|}{ CL1 (n:45) } & \multicolumn{2}{|c|}{ CL2 (n:20) } & \multicolumn{2}{|c|}{ CL3 (n:61) } & \multicolumn{2}{|c|}{ CL4 (n:49) } & \multicolumn{2}{|c|}{ CL5 (n:27) } & \multicolumn{2}{|c|}{ CL6 (n:25) } & \multicolumn{2}{|c|}{ CL7 (n:23) } \\
\hline & Mean & S.D. & Mean & S.D. & Mean & S.D. & Mean & S.D. & Mean & S.D. & Mean & S.D. & Mean & S.D. \\
\hline & $\mathrm{cm}$ & $\overline{\mathrm{cm}}$ & $\mathrm{cm}$ & $\mathrm{cm}$ & $\mathrm{cm}$ & $\mathrm{cm}$ & $\mathrm{cm}$ & $\overline{\mathrm{cm}}$ & $\mathrm{cm}$ & $\overline{\mathrm{cm}}$ & $\mathrm{cm}$ & $\overline{\mathrm{cm}}$ & $\mathrm{cm}$ & $\mathrm{cm}$ \\
\hline Height & 171.5 & 5.2 & 167.9 & 4.3 & 163.2 & 5.8 & 175.5 & 5.3 & 169.3 & 5.8 & 171.3 & 5.1 & 171.3 & 4.7 \\
\hline Shoulder length & 44.2 & 2.6 & 43.1 & 2.5 & 41.2 & 2.2 & 44.4 & 2.0 & 42.0 & 1.6 & 42.1 & 2.6 & 42.0 & 2.1 \\
\hline Shoulder seam & 13.6 & 0.9 & 13.3 & 1.1 & 13.2 & 1.0 & 13.6 & 0.8 & 13.3 & 0.8 & 13.7 & 1.1 & 13.3 & 1.0 \\
\hline 頸付根囲[45] & 42.7 & 2.8 & 42.6 & 1.9 & 40.1 & 1.4 & 42.5 & 1.8 & 40.7 & 1.7 & 39.5 & 1.5 & 40.5 & 2.0 \\
\hline Chest girth & 97.2 & 6.9 & 98.5 & 4.4 & 90.5 & 4.4 & 98.6 & 5.9 & 93.4 & 3.6 & 88.3 & 3.6 & 91.0 & 3.4 \\
\hline Waist girth & 88.4 & 9.2 & 90.8 & 6.8 & 79.8 & 6.5 & 87.0 & 7.4 & 80.8 & 6.0 & 75.4 & 5.3 & 77.7 & 4.5 \\
\hline Abdomen girth & 88.9 & 9.0 & 91.1 & 7.0 & 82.6 & 6.2 & 88.2 & 6.9 & 82.5 & 5.1 & 77.9 & 5.8 & 80.2 & 4.5 \\
\hline Hip girth & 98.3 & 5.8 & 97.7 & 5.2 & 90.8 & 3.8 & 98.3 & 4.5 & 92.9 & 3.0 & 90.2 & 3.4 & 92.4 & 4.2 \\
\hline 背幅 & 40.7 & 2.3 & 40.4 & 2.3 & 38.2 & 2.0 & 41.2 & 2.3 & 39.5 & 1.6 & 39.4 & 1.9 & 39.1 & 2.2 \\
\hline 胸幅 & 36.5 & 1.7 & 36.7 & 2.1 & 34.8 & 1.7 & 37.2 & 1.6 & 35.5 & 1.3 & 34.6 & 1.3 & 35.6 & 1.9 \\
\hline Waist length & 49.4 & 3.3 & 48.7 & 1.7 & 46.1 & 2.6 & 49.7 & 2.8 & 46.3 & 2.7 & 48.0 & 2.3 & 46.5 & 1.9 \\
\hline Front waist length & 46.2 & 3.9 & 46.8 & 5.1 & 40.4 & 4.3 & 45.8 & 3.9 & 40.9 & 3.6 & 40.1 & 4.4 & 40.7 & 2.6 \\
\hline \multirow[t]{2}{*}{ Shoulder slope } & 22.0 & 4.0 & 19.0 & 3.1 & 21.9 & 4.0 & 20.2 & 3.3 & 20.2 & 3.0 & 21.8 & 4.2 & 18.6 & 3.5 \\
\hline & $\mathrm{kg}$ & $\mathrm{kg}$ & $\mathrm{kg}$ & $\mathrm{kg}$ & $\mathrm{kg}$ & $\mathrm{kg}$ & $\mathrm{kg}$ & $\mathrm{kg}$ & $\mathrm{kg}$ & $\mathrm{kg}$ & $\mathrm{kg}$ & $\mathrm{kg}$ & $\mathrm{kg}$ & $\mathrm{kg}$ \\
\hline Body weight & 72.8 & 10.0 & 59.3 & 27.6 & 54.2 & 15.5 & 72.9 & 12.1 & 63.7 & 4.5 & 51.9 & 18.2 & 62.0 & 5.3 \\
\hline
\end{tabular}



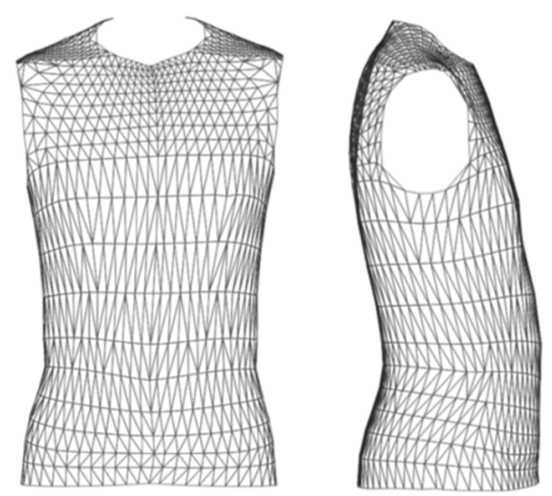

Fig. 6 The average model of the 250 subjects

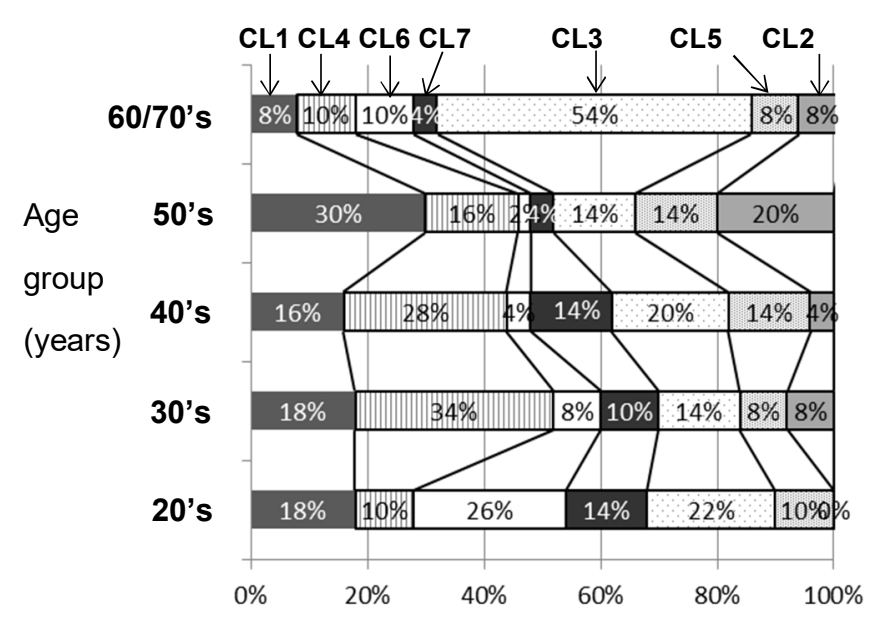

Fig. 7 The frequency of occurrence of each cluster

20s. CL7 represented good posture with a developed chest and a tight waist with muscle. The frequency of CL7 is not high, although CL7 existed in every age group. Cluster 3 (CL3) had a round back and front shoulder, and the subjects in CL3 were short. CL3 comprised less than $22 \%$ among men in the 20s to 50 s, while it comprised $54 \%$ in the group of men in their $60 / 70$ s. CL3 is a body type that was particularly common among elderly people. In cluster 5 , the trunk was inclined backward and the stomach was protruding. Cluster 2 (CL2) had a more inclined backward trunk and more protruded stomach, and is considered to be the so-called metabolism type. The frequency of CL2 reached $20 \%$ among men in their 50s, while no one was classified as CL2 among men in their 20s.

People tend to have a specific image of the body shape of men by age. The frequency was different, but in fact each body type was seen in each age group. In the design of clothes, it should be noted that there are people with various body types regardless of age.

\subsection{Application to pattern design}

This body classification system is considered to be applicable to real/virtual dress dummy design and for a made-to-measure system, and should provide more useful information for apparel manufacturers. The average models of each cluster were expressed by 3D coordinates, and are directly applicable for making dress dummies or mannequins.

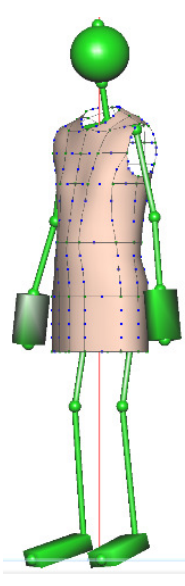

CL1

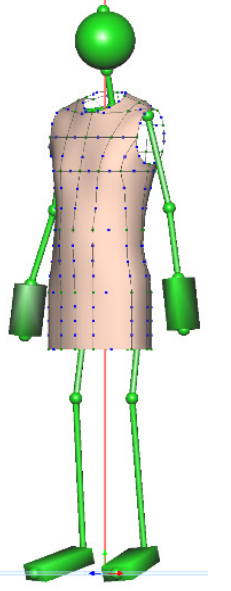

CL4

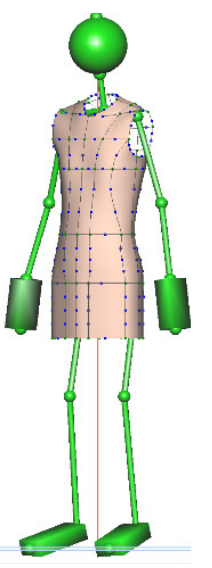

CL6

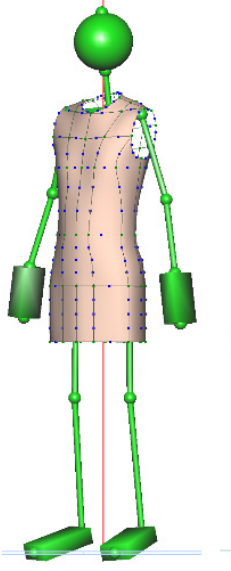

CL7

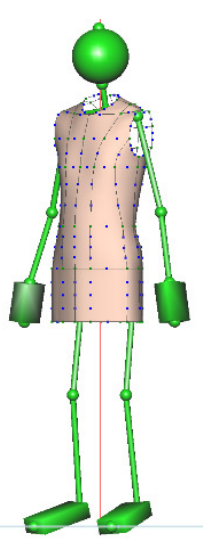

CL3

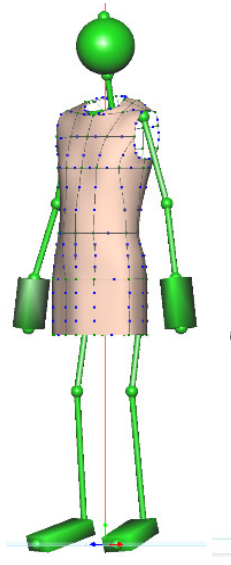

CL5

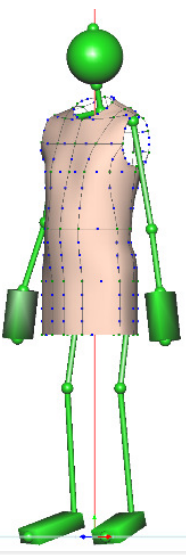

CL2

Fig. 8. The digital virtual dress dummies that had been transformed from the 7 clusters expressed in OBJ data in the 3D Apparel CAD system called "Pattern Magic II" 
In the Japan Industrial Standards, body shape is classified according to the difference between the girths of the chest and waist. Also, there are few variations in the shape of dress dummies. As a result, it is difficult to design clothes that fit various body shapes. By creating dress dummies that reflect the results of the body classification system developed in this study, it seems possible to design garment patterns that will better fit customers. The average models of the 7 clusters were expressed in OBJ data format; the data were inputted into the 3D Apparel CAD system called "Pattern Magic II" (Toray ACS, Tokyo), and transformed into digital virtual dress dummies (Fig.8). A tight-fitting film that covers the entire body in one piece is automatically generated on the body surface. Then, the neck line, armhole line, center front line, center back median line, waist line, hip line, shoulder seam line and side seam line are drawn on the film as cutting lines. The film is cut into several pieces, and each piece is developed into a flat surface in the CAD system. The body surface of each average model can be used to design a master pattern for each body type. Based on the three-dimensional measurement of a customer's body and its analysis, the master pattern of the appropriate body type is selected. Then, the master pattern is slightly adjusted according to the customer's body measurements. A remarkable change in the shape of the master pattern would not be necessary. Remarkable changes in the shape of the pattern would cause major changes in the silhouette and image, and would also cause the problem that it cannot be sewn in a factory. With the above-mentioned procedure, the author believes that a pattern of clothes more suitable for individual's body can be obtained. Similar studies have been conducted on the upper body of women [10] and on the lower body of men and women for pants design [11]. In the next study, the usefulness of this made-to-measure system will be verified.

\section{References}

[1] Ministry of Economy, Trade and Industry, http://www.meti.go.jp/committee/kenkyukai/seizou/apparel_supply/pdf/report01_03_00.pdf., accessed 2017.

[2] Shimosaka, C. and Matsuyama, Y.," A Research of Mass Customization of Apparel for Consumer Satisfaction" Otsuma Women's University bulletin. Home economics, Vol. 43, pp.105-106. 2007

[3] Ashdown, P. S. and Dunne, L." A study of Automated Custom fit: Readiness of the technology for the apparel industry", Clothing and Textile Researdh Journal, Vol.24, No2, pp.121-136, 2006

[4] Watanabe, K. "Analysis of three-dimensional torso shape and bodice pattern shape of young Japanese Women", Proceedings of Asian Workshop on 3D Body Scanning Technologies, pp.116122., 2012

[5] Japanese Industrial Standards Committee," Glossary of terms used in body measurements for clothing", Japanese Standards Association, 2010

[6] Mochimaru, M. and Kouchi M." Statistics for 3D human body forms", SAE Digital Human Modeling for Design and Engineering International Conference and Exposition, 2000, 2000

[7] Mochimaru, M. and Kouchi, M. "Statistics of 3-D body shapes using PCA or MDS and their applications", 17th World Congress on Ergonomics (IEA 2009), 2009

[8] Watanabe, K.,Takabu,H., "Analysis of three-dimensional torso shape and bodice pattern of elderly Japanese women", Proceedings of 3D Body Scanning Technologies, 2013

[9] Watanabe, K., \& Matsuyama, Y., "Angles and Length on the Lateral Silhouette as the Inputs to Apparel Pattern Drafting for Japanese Elderly Women", ITAA Proceedings 2002 Research Posters, pp.43., 2002

[10] Watanabe, K.," Body classification of women in their 20s to 70 s for virtual body design", Proceedings of annual Conference of Japan Research Association for Textile End-Uses 2017, 2017

[11] Watanabe, K., Mori, Y. et al.," Analysis of three-dimensional shape of lower body among Japanese women for designing lower body dress dummy for trousers", Proceedings of annual Conference of Japan Research Association for Textile End-Uses 2017, 2017

\footnotetext{
*watanaba@kyoto-wu.ac.jp; +81- 75-531-7162; http://www.kyoto-wu.ac.jp/english/index.html
} 